\section{Could a healthy diet attenuate COPD risk in smokers?}

\author{
Raphaëlle Varraso, ${ }^{1,2}$ Seif 0 Shaheen ${ }^{3}$
}

Let food be thy medicine and medicine be thy food.

Hippocrates

The predominant risk factor for COPD in high-income countries is cigarette smoking, ${ }^{1}$ but not all smokers develop $\mathrm{COPD}^{2}$ It has been hypothesised that a diet rich in antioxidants may counteract the deleterious oxidant effects of smoking and hence prevent COPD. ${ }^{3}$ Although most studies that have linked dietary antioxidant foods and nutrients to COPD have been cross-sectional, ${ }^{3}$ a few cohort studies have also reported associations between higher intake and a slower decline of lung function or a lower incidence of COPD. ${ }^{4-7}$

In Thorax, ${ }^{8}$ using longitudinal data from 44335 men in the Cohort of Swedish Men, Kaluza et al investigated the association between total fruit and vegetable consumption and incident COPD, according to smoking habits. COPD was ascertained through patient and death registers. Briefly, after adjustment for several potential confounders, they reported a negative and significant association between fruit and vegetable intake and the risk of COPD. This finding is consistent with a large number of crosssectional studies, and with a few longitudinal studies investigating the role of fruit and vegetable intake in the aetiology of COPD-related outcomes. Addressing the potential modifying effect of smoking on the diet-COPD association could help to clarify the potential causal role of diet. The authors reported a significant interaction between smoking and dietary intake of antioxidant-rich fruits and vegetables on the risk of COPD, and showed that the beneficial dietary associations were restricted to ex-smokers and current

${ }^{1}$ INSERM U1168, Aging and Chronic Diseases, Epidemiological and Public Health Approaches, Villejuif, France; ${ }^{2}$ UVSQ, UMR-S 1168, Université Versailles StQuentin-en-Yvelines, Versailles, France; ${ }^{3}$ Centre for Primary Care and Public Health, Barts and The London School of Medicine and Dentistry, Queen Mary University of London, London, UK

Correspondence to Dr Raphaëlle Varraso, INSERM U1168, Aging and Chronic Diseases, Epidemiological and Public Health Approaches, Villejuif 94807, France; raphaelle.varraso@inserm.fr smokers. These results are in agreement with the hypothesis that smokers, who have a higher exposure to oxidants, are more likely than never smokers to benefit from dietary antioxidants. Their interpretation was that the pathogenic mechanisms leading to COPD may be different in never smokers versus ever smokers, given different levels of oxidative stress. However, the possibility that the association between dietary intakes and COPD in current smokers and ex-smokers may have resulted from residual confounding by smoking cannot be ruled out. Given that we know little about what influences smoking susceptibility, it would have been interesting if the authors had also presented 'the other side of the coin', namely whether the effects of smoking on COPD risk were larger among those with a low intake of fruits and vegetables, and smaller among those with a high intake.

The authors also investigated the association between individual food items and the risk of COPD, and reported strong negative associations between intakes of apples and pears and green leafy vegetables and COPD risk. The findings for hard fruit are in keeping with previous observations. ${ }^{579} \mathrm{~A}$ strength of the paper was that the authors derived two dietary scores based on diet quality, and adjusted the analyses for these diet scores to account for all other dietary factors that might confound the relation between fruit and vegetable intake and COPD. The authors were thus able to conclude that a higher intake of fruits and vegetables was beneficial, independently of other components of a healthy/unhealthy diet. A huge body of nutritional epidemiology research in recent decades has led to a focus on diet quality, ${ }^{10}$ and several dietary scoresbased on prevailing hypotheses and guidance about the role of diet in disease prevention-have been developed to evaluate the healthiness of individual diets. Among these dietary scores, adherence to the recent evidence-based Alternate Healthy Eating Index 2010 (AHEI-2010) has been reported to be associated with a lower risk of COPD. ${ }^{11}$ In line with the AHEI-2010 dietary score, researchers from the Harvard Chan School of Public Health have proposed 'The Healthy Eating Plate' as a guide for creating healthy and balanced meals, with a special focus on diet quality. The first message of this 'healthy plate' is that half of our plate should comprise fruits and vegetables, which resonates with the current report of Kaluza et al. ${ }^{8}$

Besides diet per se, physical activity and body composition have also been suggested as aetiological factors for respiratory diseases. Of course, these factors are intrinsically linked to one another. Since a healthy diet (high in fruits and vegetables) is clearly associated with other healthy lifestyles, as the data clearly demonstrate, residual confounding of dietary associations remain a possibility, even after adjusting for these other risk factors in the way that they were measured. These inter-relationships between obesity, diet and physical activity raise methodological challenges in epidemiological studies, but these can now be addressed using novel approaches from the field of causal inference. $^{12}$ For instance, we have recently underlined the likely mediating role of body mass index in the diet-asthma association, ${ }^{13}$ and how mediation analyses in the counterfactual framework may help to disentangle direct and indirect effects. Another methodological challenge is posed by time-dependent confounding; it has been widely demonstrated that, in the presence of such confounding, standard methods of analysis may be biased. ${ }^{12}$ In longitudinal epidemiological studies, these complex situations of time-dependent confounding can be addressed using approaches such as marginal structural models $^{14}$ or the g-formula. ${ }^{15}$ Future research on diet and COPD will need to address these methodological challenges and take into account the complex interrelationships between diet, body composition and physical activity in order to draw more robust conclusions.

In the paper from Kaluza $e t a l,{ }^{8}$ the use of Swedish registries for COPD morbidity and mortality is a potential strength that is expected to lead to complete ascertainment of diagnosed cases, but it is also a limitation, for two reasons. First, reliance on doctor diagnosis may lead to underdiagnosis and misclassification of COPD. For example, there may be a preferential tendency to diagnose asthma rather than COPD in never smokers. The preferred, gold standard, definition of COPD in epidemiological studies is based on objective postbronchodilator spirometry. ${ }^{16}$ Second, COPD is now recognised as a more complex syndrome with concomitant comorbidities, the so-called 'comorbidome. ${ }^{17}$ As is true for asthma, a new challenge in COPD is to identify relevant 
and novel phenotypes. ${ }^{18}$ In recent years, there has been an emerging consensus that asthma and COPD often overlap, and the term 'asthma-COPD overlap syndrome' (ACOS) is increasingly applied to the condition in which a person has clinical features of both asthma and COPD. ${ }^{19}$ One of the major obstacles to understand the causes of respiratory diseases, and to improve treatment, is the failure to understand the underlying disease mechanisms in individuals with different phenotypes. ${ }^{20}$ A challenge for population-based studies aimed at identifying nutritional causes of COPD or asthma is to try to focus on truly homogeneous groups rather than heterogeneous groups of patients with 'COPD' or 'asthma', since it is possible that the influence of diet might differ across these different respiratory phenotypes. While diet appears to be a risk factor for the onset of COPD when considered as a whole, little is known regarding its association with different COPD phenotypes, ACOS or exacerbations.

Although efforts to prevent COPD should continue to focus on smoking cessation, these latest prospective findings suggest that a healthy diet (and a high intake of fruits and vegetables in particular) may have the potential to prevent COPD in smokers. While purists would say that, given the high probability of confounding in observational studies, we should wait for definitive evidence from a randomised controlled trial to confirm causality, it could be argued that there is nothing to be lost by acting now. We would argue that clinicians should consider the potential benefits of a healthy diet in promoting lung health, and advocate optimising intake of fruits and vegetables, especially in smokers who are unable to stop smoking.

\section{Competing interests None declared.}

Provenance and peer review Commissioned; externally peer reviewed.

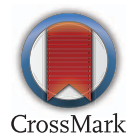

To cite Varraso R, Shaheen SO. Thorax 2017;72:491-492.

Received 6 December 2016

Revised 4 January 2017

Accepted 6 January 2017

Published Online First 27 January 2017

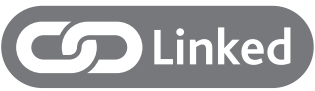

http://dx.doi.org/10.1136/thoraxjnl-2015-207851

Thorax 2017;72:491-492.

doi:10.1136/thoraxjnl-2016-209608

\section{REFERENCES}

1 Speizer FE, Tager IB. Epidemiology of chronic mucus hypersecretion and obstructive airways disease. Epidemiol Rev 1979;1:124-42.

2 Fletcher C, Peto R, Tinker R, et al. The Natural History of Chronic Bronchitis and Emphysema. Oxford; Oxfort uni, 1976.

3 Berthon BS, Wood LG. Nutrition and respiratory health-feature review. Nutrients 2015;7:1618-43.

4 Walda IC, Tabak C, Smit H, et al. Diet and 20-year chronic obstructive pulmonary disease mortality in middle-aged men from three European countries. Eur J Clin Nutr 2002;56:638-43.

5 Miedema I, Feskens EJ, Heederik D, et al. Dietary determinants of long-term incidence of chronic nonspecific lung diseases. The Zutphen Study. Am J Epidemiol 1993;138:37-45.

6 Butler LM, Koh W-P, Lee H-P, et al. Dietary fiber and reduced cough with phlegm: a cohort study in Singapore. Am J Respir Crit Care Med 2004;170:279-87.
7 Butland BK, Fehily AM, Elwood PC. Diet, lung function, and lung function decline in a cohort of 2512 middle-aged men. Thorax 2000;55:102-8.

8 Kaluza J, Larsson S, Orsini N, et al. Fruit and vegetable consumption and risk of COPD: a prospective cohort study of men. Thorax 2017 2017:72:500-9.

9 Tabak C, Smit HA, Heederik D, et al. Diet and chronic obstructive pulmonary disease: independent beneficial effects of fruits, whole grains, and alcohol (the MORGEN study). Clin Exp Allergy 2001;31:747-55.

10 Willett WC, Stampfer MJ. Current evidence on healthy eating. Annu Rev Public Health 2013;34:77-95.

11 Varraso R, Chiuve SE, Fung TT, et al. Alternate Healthy Eating Index 2010 and risk of chronic obstructive pulmonary disease among US women and men: prospective study. BMJ 2015;350:h286.

12 Robins JM, Hernán MA, Brumback B. Marginal structural models and causal inference in epidemiology. Epidemiology 2000;11:550-60.

13 Li Z, Rava M, Bédard A, et al. Cured meat intake is associated with worsening asthma symptoms. Thorax 2017:72:206-12.

14 Dumas O, Le Moual N, Siroux V, et al. Work related asthma. A causal analysis controlling the healthy worker effect. Occup Environ Med 2013;70:603-10.

15 Bédard A, Serra-Pons I, Dumas 0, et al. Time-dependent associations between body composition, physical activity and current asthma in women using marginal structural models. $\mathrm{Am}$ J Epidemiol, In press.

16 Rabe KF, Hurd S, Anzueto A, et al. Global strategy for the diagnosis, management, and prevention of chronic obstructive pulmonary disease: GOLD executive summary. Am J Respir Crit Care Med 2007; 176:532-55

17 Divo M, Cote C, de Torres JP, et al. Comorbidities and risk of mortality in patients with chronic obstructive pulmonary disease. Am J Respir Crit Care Med 2012;186:155-61.

18 Han MK, Agusti A, Calverley PM, et al. Chronic obstructive pulmonary disease phenotypes: the future of COPD. Am J Respir Crit Care Med 2010:182:598-604.

19 Postma DS, Rabe KF. The asthma-COPD overlap syndrome. N Engl J Med 2015;373:1241-9.

20 Gibson PG, McDonald VM. Asthma-COPD overlap 2015: now we are six. Thorax 2015;70:683-91. 